\title{
Study and Analysis of the Magneto-Mechanical Behavior of Smart Composite Sandwich Beam in Elastomer
}

\author{
Ahmed Tidjani SETTET*, Salah AGUIB****, Abdelkader NOUR*, Nassim ZERROUNI* \\ *Dynamic Motors and Vibroacoustic Laboratory, Department of Mechanical Engineering, M'Hamed Bougara University \\ of Boumerdes, Algeria, E-mail: s.aguib@univ-boumerdes.dz \\ **Laboratory of Condensed Matter Physics, University of Nice-Sophia Antipolis, CNRS, UMR 7336, 28 avenue Joseph \\ Vallot, 06100 Nice, France
}

cross $^{\text {ref }}$ http://dx.doi.org/10.5755/j01.mech.25.4.22713

\section{Introduction}

Composite materials are materials with high mechanical properties; its mechanical properties are outperforming those of each of its components taken separately. Composite materials are used on the day of today in all areas and especially in high technology. Materials for which rheological properties can be controlled by the application of a magnetic field are called magnetorheological materials (MR) [1-3]. They belong to the class, more widely defined, active materials since they can respond to changes in their environment, changes brought here by semiconductors and appropriate control algorithms. Such materials can be used directly in devices or incorporated into composites to create advanced composite structures, making their multiple applications in the automotive, aerospace and electronics industries. The magnetorheological elastomers (MRE) consist of ferromagnetic particles (generally of micrometric size) dispersed in a silicone elastomeric matrix [4-6]. Their low response time (on the order of one millisecond), their continuously controllable properties, and their ability to withstand wide variations in rigidity make MRE attractive for potential applications in aerospace, automotive, civil engineering or in electrical engineering [7-11]. Examples include adaptive lenses, interactive man-machine interfaces, damping devices and variable stiffness supports. Bingbing Kang et al. [12] have studied the influence of parameter modifications on nonlinear mechanical properties. On this basis, they designed a Ruzicka vibration damping model high static-low-dynamic and studied its vibration isolation characteristics. In recent years, the scientific community has focused on the knowledge of rheological behavior materials. Sobhan et al. [13] used the asymptotic homogenization method to estimate the elastic properties as well as the tensile, flexural and torsional stiffness of single-layer graphene sheets (SLGS). To this end, asymptotic homoge-nization of a reinforced composite is developed for mode-ling of SLGS by assuming that the covalent bond between carbon atoms can be represented by reinforcements. Based on the above works, the static study and characteristics of bending sandwich beam were investigated in this present paper. By this way, we have studied the influence of the magnetic field and the length of the adaptive beam on the amplitude of transverse displacement; we have therefore developed an adaptive structure to dampen the amplitude of the displacements in the form of high energy dissipation.
2. Mathematical modeling of bending mechanical behavior

In this section, we consider a three-layer beam simply supported (Fig.1); the latter is exposed to a uniformly distributed magnetic field (Fig.2). The mechanical and geometrical characteristics of each element of the beam are represented by Fig.3.

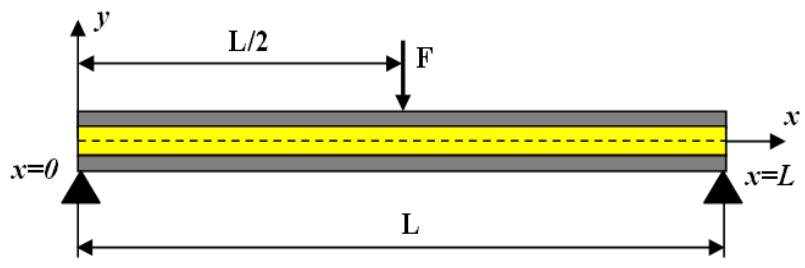

Fig. 1 Simply supported three-layered sandwich beam

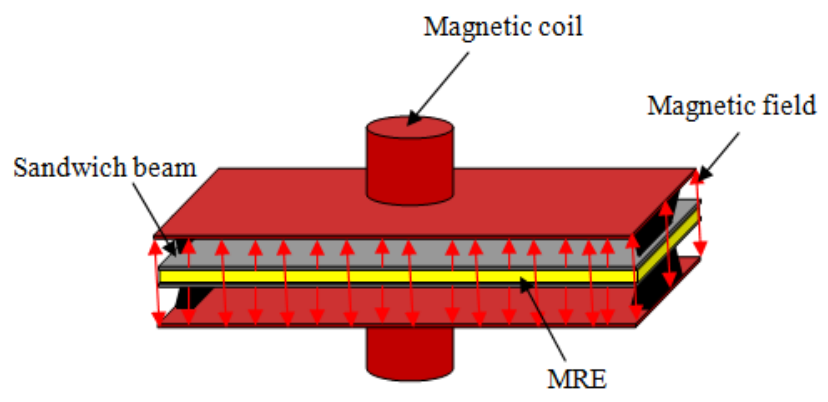

Fig. 2 Schematic of the sandwich beam with elastomer magnetorheological

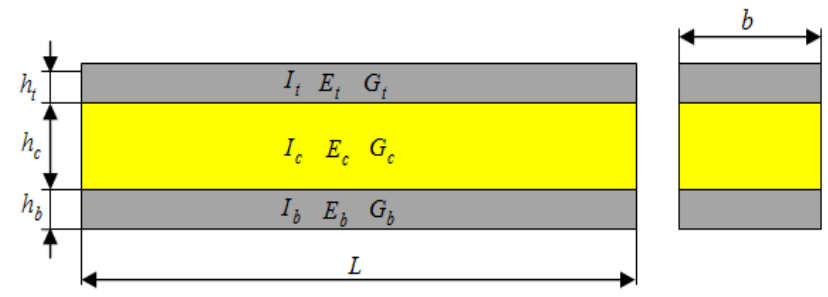

Fig. 3 Geometrical Schematic of a sandwich beam elements

The boundary conditions are given as follows:

$$
x=0 \Rightarrow\left\{\begin{array}{l}
M=0 \\
w=0 \\
Q=0
\end{array}, x=L \Rightarrow\left\{\begin{array}{l}
M=0 \\
w=0 . \\
Q=0
\end{array}\right.\right.
$$


beam without resorting to complex theories of higher order, the Nilsson model, taking some of the principles from the Timoshenko theory will be used. However, Nilsson works were interested in a sandwich structure where skins are homogeneous materials.

In this case, if the equilibrium equation, written as a function of the longitudinal elasticity and the shear moduli of the beam, is given by:

$$
\frac{d M(x)}{d x}-(G A)_{e q}\left(\phi_{x}+\frac{d w}{d x}\right)=0
$$

wherein the expression of the transverse shear angle along the axis is given by:

$$
\frac{d \phi(x)}{d x}=\frac{M(x)}{(E I)_{e q}}=\frac{1}{2(E I)_{e q}}\left(F-F_{m}\right) x
$$

with: $F_{m}=\frac{B^{2} b h_{j}}{\mu_{e j}} \frac{\partial w_{j}^{2}}{\partial x^{2}}$ by integrating Eq. (2) we deduce the following expression :

$$
\phi(x)=\frac{1}{4(E I)_{e q}}\left(F-\frac{B^{2} b h_{j}}{\mu_{e j}} \frac{\partial w_{j}^{2}}{\partial x^{2}}\right) x^{2}+k,
$$

the symmetry of the beam requires:

$$
\begin{aligned}
& \phi(x)=\frac{1}{4(E I)_{e q}}\left(F-\frac{B^{2} b h_{j}}{\mu_{e j}} \frac{\partial w_{j}^{2}}{\partial x^{2}}\right) x^{2}- \\
& -\frac{L^{2}}{16(E I)_{e q}}\left(F-\frac{B^{2} b h_{j}}{\mu_{e j}} \frac{\partial w_{j}^{2}}{\partial x^{2}}\right),
\end{aligned}
$$

by substituting Eq. (5) into Eq. (2) we obtain:

$$
\begin{aligned}
& \frac{d w}{d x}=-\frac{1}{4(E I)_{e q}}\left(F-\frac{B^{2} b h_{j}}{\mu_{e j}} \frac{\partial w_{j}^{2}}{\partial x^{2}}\right) x^{2}+ \\
& +\frac{L^{2}}{16(E I)_{e q}}\left(F-\frac{B^{2} b h_{j}}{\mu_{e j}} \frac{\partial w_{j}^{2}}{\partial x^{2}}\right)+ \\
& +\frac{1}{2(G A)_{e q}}\left(F-\frac{B^{2} b h_{j}}{\mu_{e j}} \frac{\partial w_{j}^{2}}{\partial x^{2}}\right),
\end{aligned}
$$

finally, by the integration of Eq. (6), the response of the magneto-visco-elastic behavior of the Timoshenko beam is given in the form:

$$
\begin{aligned}
& w(x)=-\frac{1}{12(E I)_{e q}}\left(F-\frac{B^{2} b h_{j}}{\mu_{e j}} \frac{\partial w_{j}^{2}}{\partial x^{2}}\right) x^{3}+ \\
& +\frac{L^{2}}{16(E I)_{e q}} F x-\frac{L^{2}}{16(E I)_{e q}} \frac{B^{2} b h_{j}}{\mu_{e j}} \frac{\partial w_{j}^{2}}{\partial x^{2}} x+ \\
& +\frac{1}{2(G A)_{e q}}\left(F-\frac{B^{2} b h_{j}}{\mu_{e j}} \frac{\partial w_{j}^{2}}{\partial x^{2}}\right) x,
\end{aligned}
$$

where and is the bending and shear rigidity of beam respectively. The maximum deflection is given for as follows:

$$
w_{\max }\left(\frac{L}{2}\right)=-\left(\frac{L^{3}}{24(E I)_{e q}}+\frac{L}{4(G A)_{e q}}\right)\left(F-\frac{B^{2} b h_{j}}{\mu_{e j}} \frac{\partial w_{j}^{2}}{\partial x^{2}}\right),
$$

the equivalent bending rigidity of the beam is given by Eq. 9.

$$
(E I)_{e q}=\frac{1}{12} E_{c} h_{c}+E_{p}\left(\frac{1}{2} h_{c}^{2}+h_{c} h^{2}+\frac{2}{3} h^{3}\right)
$$

where: $h_{t}=h_{b}=h$ represents the thicknesses of the top and bottom skins and $E_{t}=E_{b}=E_{p}$ Young's moduli of the aluminum skins (t: top and b: bottom). by Eq. 10 .

The equivalent shear rigidity of the beam is given

$$
(G A)_{e q}=\frac{b d^{2} G_{c}}{c} \simeq b d G_{c}=A G_{c},
$$

where: $G_{c}$ is the shear modulus of the elastomer core and $A$ is the section of the beam ( $c$ : core). The complex shear modulus of the magneto-rheological material can be expressed as $G_{c}=G^{\prime}+i G^{\prime \prime}$, where $G^{\prime}$ and $G^{\prime \prime}$ are loss and storage moduli, respectively, and described as a polynomial function of the magnetic field intensity $B$, for expressed magneto-rheological material, as:

$$
\begin{aligned}
& G^{\prime}=-3.369 B^{2}+4.9975 \times 10^{3} B+0.873 \times 10^{6}, \\
& G^{\prime \prime}=-0.9 B^{2}+0.81240 \times 10^{3} B+0.8550 \times 10^{6} .
\end{aligned}
$$

\section{Analytical analysis}

The mechanical and geometrical characteristics of the beam are given in (Table 1).

The variation in the bending through the distance between supports is given in Fig. 4, for different values of magnetic field intensity. It is observed that the deflection of the beam is strongly dependent on the magnetic field intensity and its value decreases with the increase of the magnetic field intensity. For $L=250 \mathrm{~mm}$ the value of deformation is $26.729 \mathrm{~mm}$ for a value of $B=0.1 \mathrm{~T}$ and $21.423 \mathrm{~mm}$ for $B=0.3 \mathrm{~T}$ and a value of $19.765 \mathrm{~mm}$ for $B=0.5 \mathrm{~T}$.

The influence of the stiffness in bending $E I$ and in shear on the maximum deflection of the beam is illustrated in Figs. 5 and 6. These figures show that the maximum deflection $w_{\max }$ depends essentially on stiffness. This effect is particularly remarkable and important as a function of the variation of magnetic field intensity, so when the value of $B$ increases the stiffness in bending and in shear increases, unlike the maximum deflection which decreases.

For comparison, the results obtained show clearly that the influence of the shear stiffness is less than the influence of bending stiffness on the beam behavior. 
Table 1

Mechanical, electrical and geometrical properties of the beam

\begin{tabular}{|l|c|c|c|c|}
\hline Material properties & $\rho, \mathrm{kg} / \mathrm{m}^{3}$ & $E, \mathrm{MPa}$ & $v$ & $\mu_{e j}, \mathrm{Hm}^{-1}$ \\
\hline Aluminum skins & 2800 & 72000 & 0.33 & $1.2566650 \times 10^{-6}$ \\
\hline Elastomer & & & 0.45 & \\
\hline Geometrical characteristics of the top (t) and bottom (b) skins \\
\hline$b, \mathrm{~mm}$ & $L, \mathrm{~mm}$ & $h_{t}, \mathrm{~mm}$ & $h_{b}, \mathrm{~mm}$ & \\
\hline 30 & 300 & 1 & 1 & \\
\hline Geometrical characteristics of the elastomer & & \\
\hline$b, \mathrm{~mm}$ & $L, \mathrm{~mm}$ & $h_{c}, \mathrm{~mm}$ & & \\
\hline 30 & 300 & 2 & & \\
\hline
\end{tabular}

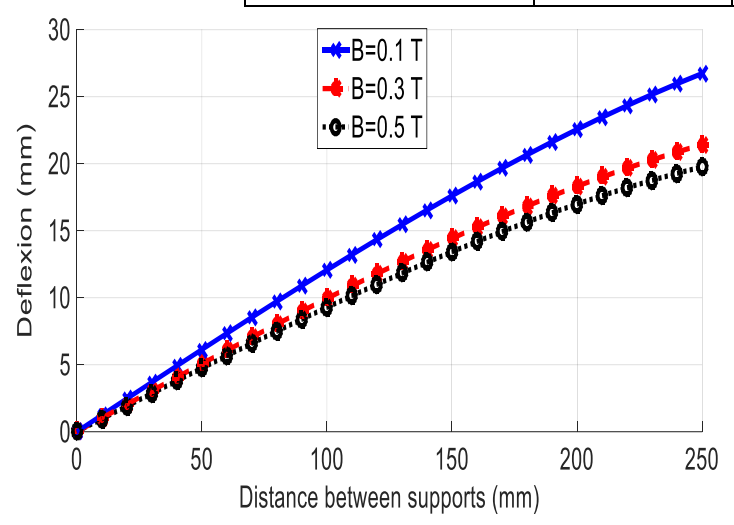

Fig. 4 Deflection obtained by finite elements for different values of magnetic field intensity

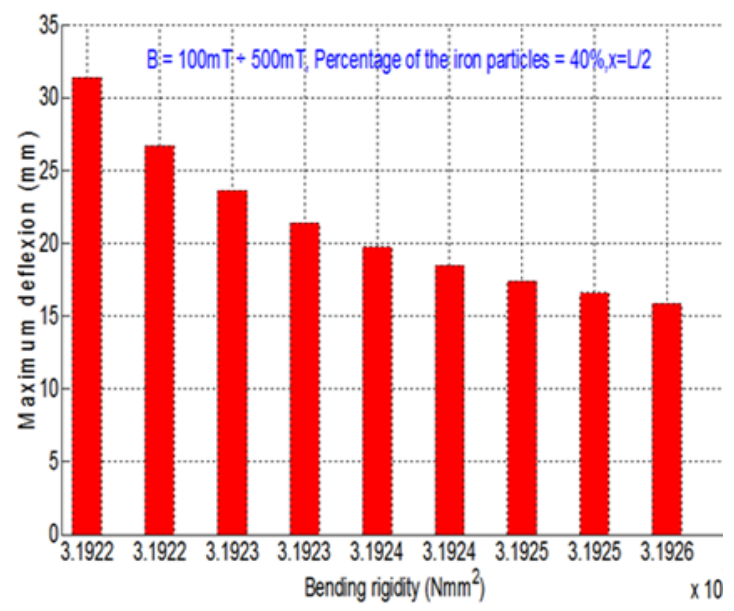

a

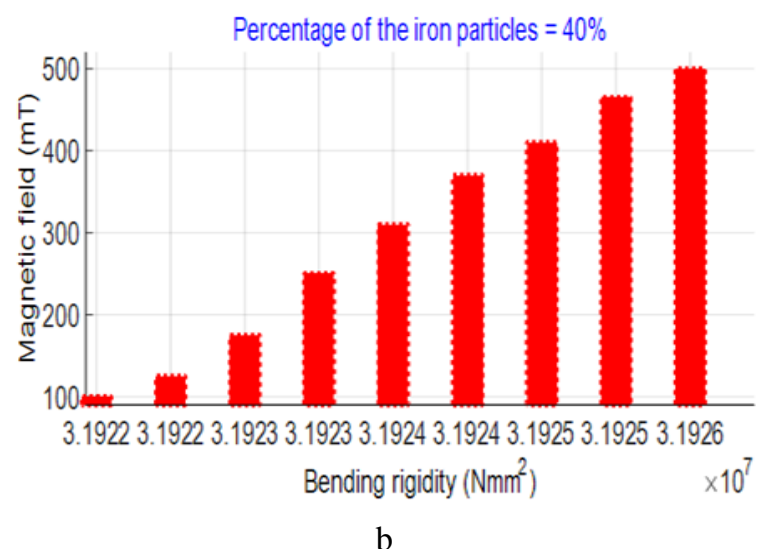

Fig. 5 Variation of the equivalent bending stiffness of the sandwich beam, a) maximum deflection b) magnetic field

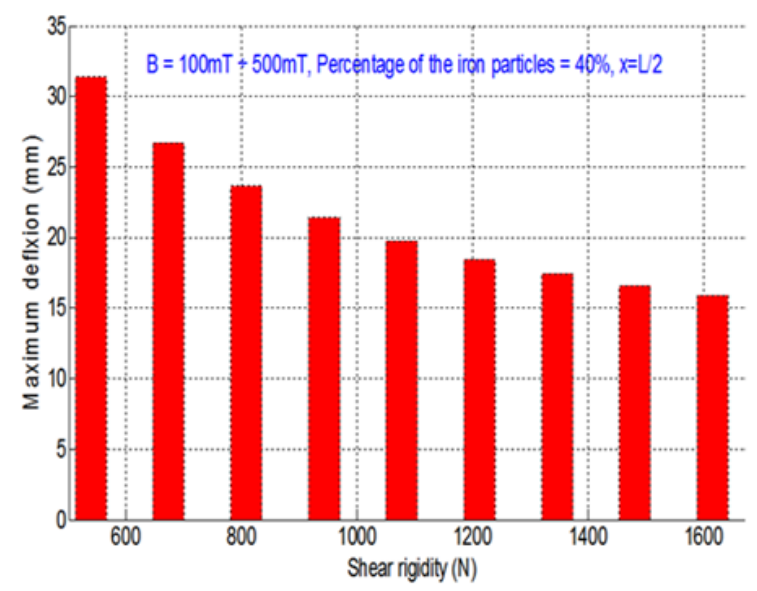

a

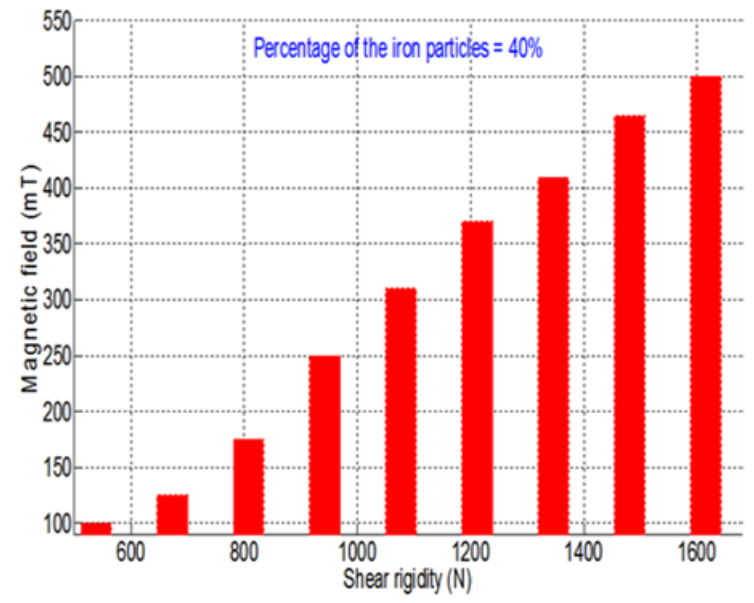

b

Fig. 6 Variation of the equivalent shear stiffness of the sandwich beam, a) maximum deflection b) magnetic field

\section{Numerical simulation}

The simulation of the structures by finite elements is performed using the Abaqus software. The element used is a 2-D element, CPS4 type with four nodes (Fig. 7). The sandwich material is modeled by three structures, two isotropic elastic structures corresponding to the aluminum skins, and a MRE core. The skins are characterized by the Young's modulus, Poisson's ratio and density, given in Table 1. The sandwich beam model realized in Abaqus is given in Fig. 8.

Fig. 9 shows the deflection of the composite material structure for different values of the magnetic field intensity. 


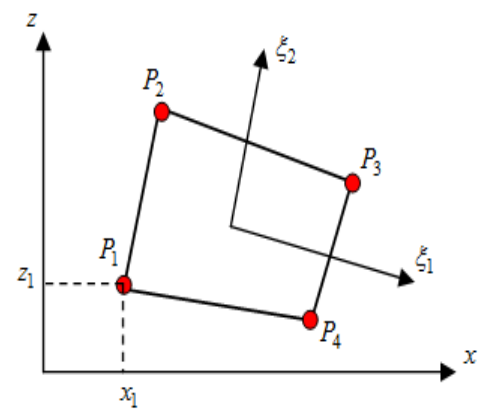

The results of meshing of the beam in Abaqus are given in Table 2.

Table 2

Problem size of meshing by FEM

\begin{tabular}{|l|l|}
\hline Number of elements & 16000 \\
\hline Number of nodes & 21105 \\
\hline Number of nodes defined by the user & 21105 \\
\hline Total number of variables in the model & 63315 \\
\hline
\end{tabular}

Fig. 7 Mesh element in Abaqus: 2D type CPS4

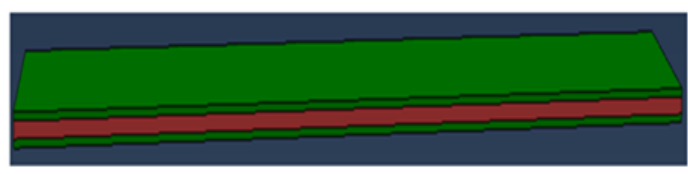

a

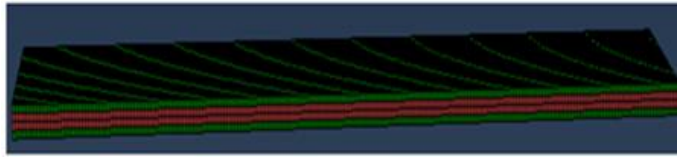

b

Fig. 8 Sandwich beam model in Abaqus a) without mesh b) with mesh

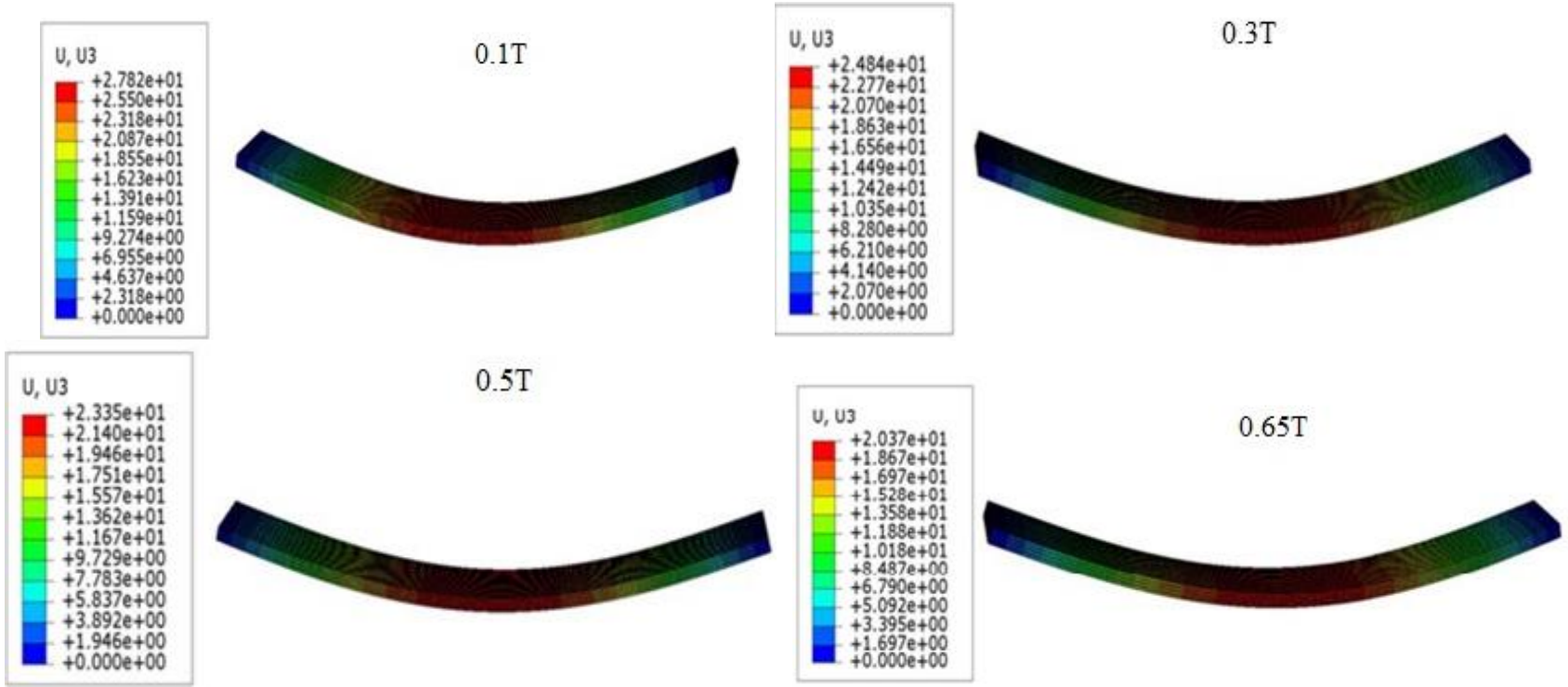

Fig. 9 Deflection obtained by finite elements for different values of the magnetic field intensity

\section{Comparison of the deflections obtained theoretically and by finite elements}

Table 3 reports the values of deflection obtained analytically and those obtained by finite elements as well as the differences. In the case of $B=0.1 T$ the difference between the results obtained analytically and those ob- tained by FEM does not exceed $6.25 \%$ for the gap does not exceed $7 \%$ and for the deviation does not exceed $9 \%$, while the gap $12.25 \%$ to reach a high attraction force. This difference can be attributed to the side effects of the magnetic field on the behavior of MRE, which is ignored by the analytical calculation.

Comparison of deflections analytically obtained and those obtained by finite elements

\begin{tabular}{|c|c|c|c|c|c|c|c|c|c|c|c|c|}
\hline & \multicolumn{3}{|c|}{$B=0.1 T$} & \multicolumn{3}{|c|}{$B=0.3 T$} & \multicolumn{3}{|c|}{$B=0.5 T$} & \multicolumn{3}{|c|}{$B=0.65 T$} \\
\hline$x, \mathrm{~mm}$ & AN & FEM & $\Delta \%$ & $\mathrm{AN}$ & FEM & $\Delta \%$ & $\mathrm{AN}$ & FEM & $\Delta \%$ & $\mathrm{AN}$ & FEM & $\Delta \%$ \\
\hline 20 & 02.463 & 02.318 & 06.25 & 02.215 & 02.070 & 07.00 & 02.038 & 01.946 & 04.70 & 01.905 & 01.697 & 12.25 \\
\hline 40 & 04.913 & 04.637 & 05.95 & 04.417 & 04.140 & 06.69 & 04.064 & 03.892 & 04.42 & 03.798 & 03.395 & 11.87 \\
\hline 60 & 07.338 & 06.955 & 05.50 & 06.595 & 06.210 & 06.20 & 06.064 & 05.837 & 03.89 & 05.666 & 05.092 & 11.27 \\
\hline 80 & 09.725 & 09.274 & 04.86 & 08.734 & 08.280 & 05.50 & 08.027 & 07.783 & 03.13 & 07.496 & 06.790 & 10.39 \\
\hline 100 & 12.062 & 11.590 & 04.00 & 10.824 & 10.350 & 04.80 & 09.940 & 9.729 & 02.20 & 09.276 & 08.487 & 09.30 \\
\hline 120 & 14.337 & 13.910 & 03.00 & 12.910 & 12.420 & 03.95 & 11.790 & 11.670 & 01.00 & 10.994 & 10.180 & 08.00 \\
\hline 140 & 16.536 & 16.230 & 01.88 & 14.803 & 14.490 & 02.20 & 13565 & 13.620 & 00.04 & 12.636 & 11.880 & 06.36 \\
\hline 160 & 18.648 & 18.550 & 00.53 & 16.667 & 16.560 & 00.60 & 15.252 & 15.570 & 02.00 & 14.191 & 13.580 & 05.00 \\
\hline 180 & 20.659 & 20.870 & 01.00 & 18.431 & 18.630 & 01.08 & 16.839 & 17.510 & 04.00 & 15.645 & 15.280 & 02.40 \\
\hline 200 & 22.558 & 23.180 & 02.75 & 20.082 & 20.700 & 03.08 & 18.313 & 19460 & 06.26 & 16.986 & 16.970 & 00.09 \\
\hline 220 & 24.332 & 25.500 & 04.80 & 21.608 & 22.770 & 05.40 & 19.662 & 21.400 & 08.84 & 18.203 & 18.670 & 02.56 \\
\hline 250 & 26.729 & 27.820 & 04.00 & 23.634 & 24.840 & 05.00 & 21.423 & 23.350 & 09.00 & 19.765 & 20.370 & 03.00 \\
\hline
\end{tabular}




\section{Experimental analysis}

\subsection{Description of the test}

Sandwich specimens of size $200 \mathrm{~mm}$ x $35 \mathrm{~mm}$ x $4 \mathrm{~mm}$ are manufactured using two aluminum skins of $1 \mathrm{~mm}$ thickness and an elastomer core charged at $30 \%$ by ferromagnetic particles of micrometric size is inserted between the two skins (Fig. 10). The three-point bending test is carried out using a Zwick machine at $2.5 \mathrm{kN}$ (Fig. 11). The tests are carried out with a constant deformation rate of $1 \mathrm{~mm} / \mathrm{min}$ in order to be able to consider that the loading is quasi-static. The applied force and the displacements are measured by the cell of the machine.

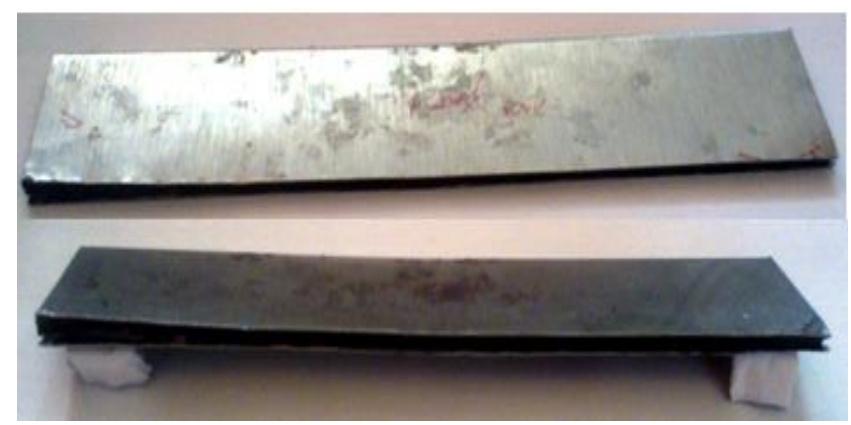

Fig. 10 MRE sandwich specimens

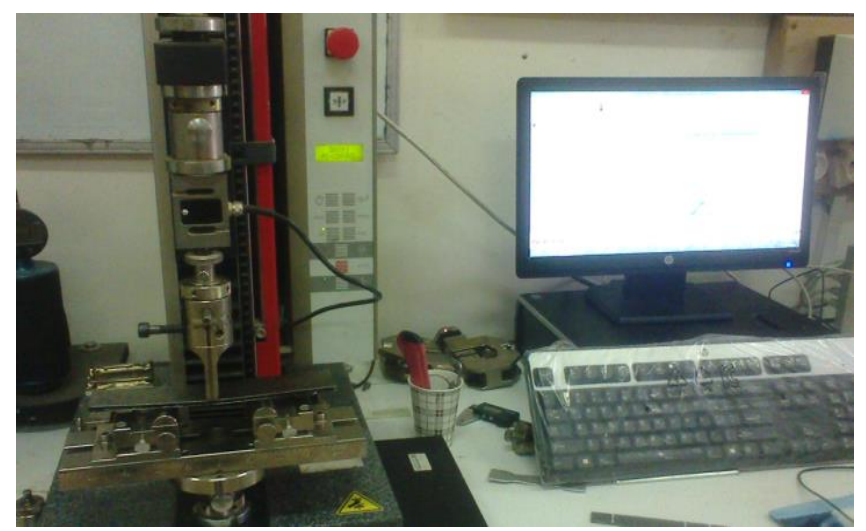

Fig. 11 Three-point bending test machine

\subsection{Interpretation of the results (Force / Displacement)}

The force-displacement curve of the beam subjected to a three-point bending load is given in Fig. 12 . From this curve, it can be seen that the breaking force is about of $115 \mathrm{~N}$ for the test of the part subjected to a magnetic field intensity of zero. On the other hand, the specimen subjected to a magnetic field intensity of $0.5 T$ is quite far from the rupture. It can be clearly seen that the rupture of the last specimen is not yet reached even for the value of the maximum applied force (around $150 \mathrm{~N}$ ) during the test.

As well as the curve in Fig. 12 shows that specimens exhibit non-linear behavior, even at small deformations. It is possible to define separate domains:

For the test piece subjected to a magnetic field intensity of $0 T$, we can observe a behavior more or less linear at the beginning of displacement and for a force value less than $20 \mathrm{~N}$, then a non-linear behavior until the rupture. On the other hand, the specimen subjected to a magnetic field intensity of $0.5 T$ presents a non-linear behavior even with practically negligible displacements.

We can observe, for a zero magnetic field, that the maximum force applied on the specimen to reach the rup- ture of the aluminum layers is $115 \mathrm{~N}$ (the aluminum layers are broken before the MRE core), then this force gradually decreases and this can be explained by the highly deformable viscoelastic behavior of the MRE, that is to say it is able to withstand very large deformations without breaking. For a magnetic field intensity of $0.5 T$ the aluminum layers are also broken at a force of $115 \mathrm{~N}$, but on the contrary, the force increases constantly and this is explained by the significant increase in the stiffness of the MRE due to the force of attraction between the ferromagnetic particles

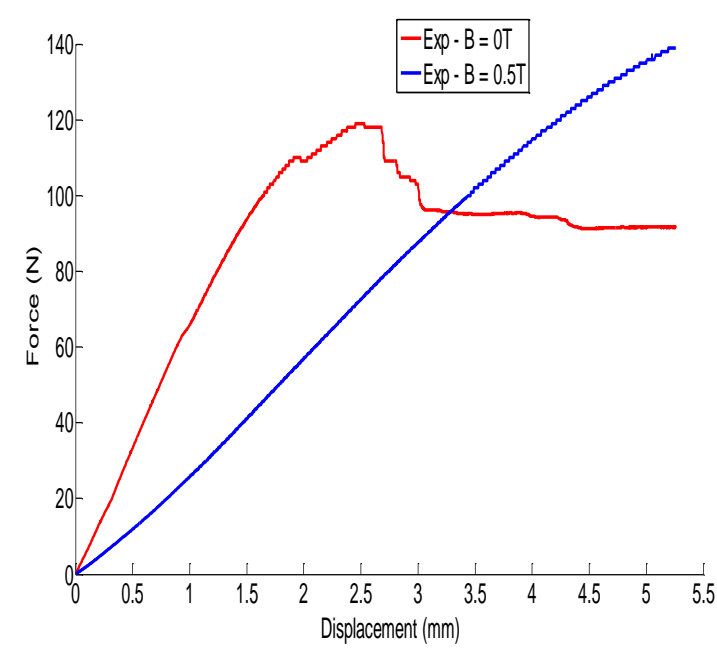

Fig. 12 Three-point bending strength/displacement curve of the sandwich beam with and without magnetic field intensity

\section{Conclusions}

The magnetic properties of MRE have been studied by several researches before, but the study on sandwich structures in MRE is new. The MRE manufactured with iron particles dispersed in a natural rubber was studied under magnetic field intensities from 0 to $0.65 T$.

The results obtained by the analytical study (section 2.1) are compared with those obtained by finite element analysis. The calculations of the deflection were performed taking into account the variation of the rheological characteristics of the MRE according to different magnetic field intensities.

The analysis of the results shows that:

Overall, the deflection of the beam decreases with the increase of the magnetic field intensity.

The results obtained by finite elements and those obtained by analytical study are in good agreement for all magnetic field intensities.

The deflection obtained numerically deviates slightly with the increase of the magnetic field intensity of: $6.25 \%$ for $B=0.1 T, 7 \%$ for $B=0.3 T, 9 \%$ for $B=0.5 T$, and $12.25 \%$ for $B=0.65 T$.

The evolutions of the deflection as a function of the length of the beam depend on several parameters: the distribution of strain energy between the skins and the core, the magnetic field intensity as well as the elastomer charging value by the iron particles.

It was shown in the Figs. 5 and 6 that the magnetic field intensity has a significant influence on the rigidity of the magnetorheological elastomer. 
A difference between the analytical values of deflection of the beam and those obtained by finite elements analysis appears for high values of the magnetic field intensity. This gap could be explained by the influence of the secondary effects of the magnetic field on certain parameters which are not taken into account by the theoretical study.

\section{References}

1. Gibson, R. F. 2010. A review of recent research on mechanics of multifunctional composite materials and structures, Composite Structures 92:2793-2810. https://doi.org/10.1016/j.compstruct.2010.05.003.

2. Mikhasev, G. I.; Altenbach, H.; Korchevskaya, E. A. 2014. On the influence of the magnetic field on the eigenmodes of thin laminated cylindrical shells containing magnetorheological elastomer, Composite Structures 113:186-196. https://doi.org/10.1016/j.compstruct.2014.02.031.

3. Nayak, B.; Dwivedy, S. K.; Murthy, K. R. K. 2014. Dynamic stability of a rotating sandwich beam with magnetorheological elastomer core, European Journal of Mechanics - A/Solids 47:143-155. https://doi.org/10.1016/j.euromechsol.2014.03.004.

4. Zhang, X.; Li, W.; Gong X. L. 2008. An effective permeability model to predict field dependent modulus of magnetorheological elastomers, Communications in Nonlinear Science and Numerical Simulation 13:19101916. https://doi.org/10.1016/j.cnsns.2007.03.029.

5. Chen, L.; Gong, X. L.; Li, W. H. 2008. Effect of carbon black on the mechanical performances of magnetorheological elastomers, Polymer Testing 27:340-345. https://doi.org/10.1016/j.polymertesting.2007.12.003.

6. Sun, T. L.; Gong, X. L.; Jiang, W. Q.; Li, J.F.; Xu, Z. B.; Li, W. H. 2008. Study on the damping properties of magnetorheological elastomers based on cispolybutadiene rubber, Polymer Testing 27:520-526. https://doi.org/10.1016/j.polymertesting.2008.02.008.

7. Li, J.; Li, Y.; Li, W.; Samali, B. 2013. Development of adaptive seismic isolators for ultimate seismic protection of civil structures, Proc. of SPIE Vol. 8692. https://doi.org/10.1117/12.2009626.

8. Usman, M.; Sung, S. H.; Jang, D. D.; Jung, H. J.; Koo, J. H. 2009. Numerical investigation of smart base isolation system employing MR elastomer, Journal of Physics: Conference Series 149:012099. https://doi.org/10.1088/1742-6596/149/1/012099.

9. Karavasilis, T. L.; Ricles, J. M.; Sause, R.; Chen, C. 2011. Experimental evaluation of the seismic performance of steel MRFs with compressed elastomer dampers using large-scale real-time hybrid simulation, Engineering Structures 33:1859-1869. https://doi.org/10.1016/j.engstruct.2011.01.032.

10. Sun, S.; Deng, H.; Yang, J.; Li, W.; Du, H.; Alici, G.
2015. An adaptive tuned vibration absorber based on multilayered MR elastomers, Smart Materials and Structures 24: 045045.

https://doi.org/10.1088/0964-1726/24/4/045045.

11. Sun, S. S.; Chen, Y.; Yang, J.; Tian, T.F.; Deng, H. X.; Li, W.H. 2014.The development of an adaptive tuned magnetorheological elastomer absorber working in squeeze mode, Smart Materials and Structures 23: 075009. https://doi.org/10.1088/0964-1726/23/7/075009.

12. Bingbing, K.; Haijun L.; Zhen, Z.; Hongyang, Z. 2018. A Study of a Ruzicka Vibration Isolator Model with High-Static-Low Dynamic Characteristic, Mechanika 24: 422-431. http://dx.doi.org/10.5755/j01.mech.24.4.20302.

13. Sobhan, S.; Masoud, T. 2018. Evaluation of Tension, Bending and Twisting Rigidities of Single-Layer Graphene Sheets by an Analytical Asymptotic Homogenization Model, Mechanika 24: 161-168. http://dx.doi.org/10.5755/j01.mech.24.2.17822.

\section{A.T. Settet, S. Aguib, A. Nour, N. Zerrouni \\ STUDY AND ANALYSIS OF THE MAGNETO- MECHANICAL BEHAVIOR OF SMART COMPOSITE SANDWICH BEAM IN ELASTOMER}

S u m m a r y

The purpose of this work is to analyze the nonlinear magneto-mechanical behavior of sandwich structures with a magnetorheological elastomer (MRE) core subjected to a permanent magnetic field. A detailed study is first carried out to characterize the mechanical behavior of these structures. The tests were carried out in three-point bending on beams of these complex materials for several distances between supports. An experimental study of the static response, is realized using a Zwick $2.5 \mathrm{kN}$ machine, allows to measure displacements as a function of force. The results deduced from the numerical simulation by the Abaqus software are compared with those obtained from the theoretical analysis. This study made it possible to show that these structures exhibit a non-linear behavior even at small deformations due to the rheological parameters which are more sensitive by the application of a magnetic field.

Keywords: composite sandwich beam, magnetomechanical behavior, magnetorheological elastomer, magnetic field.

Received March 03, 2019

Accepted August 26, 2019 